\title{
Development of process technology for the preparation of yam (Amorphophallus spp.) chips
}

\section{Arvind Kumar, Alok Nath and Pratibha Devi Sharma}

See end of the Paper for authors' affiliation

Correspondence to :

Alok Nath

Department of Post-Harvest

Technology, College of

Horticulture (S.D.A.U.), Jagudan, Mehsana (Gujarat) India

Email : alok_1001@yahoo.

com
- ABSTRACT : Yam (Amorphophallus spp.) known as Ole, Balukand, Suran or Zamikand in India represents an important food crop rich in starch. It is utilized as fresh vegetable after boiling or cooking the peeled and sliced tubers. Many value added products like chips, dehydrated slices, flour, pickles etc. can be made out of available elephant foot yam tubers. The process technology for producing chips was developed and process parameters were optimized. The prepared yam chips after pre-treatments of blanching in water and KMS $(0.5 \%)$ were subjected for drying at temperatures viz., 60,70 and $80^{\circ} \mathrm{C}$. The fried chips samples were subjected to organoleptic evaluation using 9-point hedonic scale and based on maximum average scores for different quality attributes, the best sample found was chips prepared with $\left(\mathrm{T}_{\mathrm{C} 2}\right)$ i.e. blanched with 0.5 per cent KMS and dried at $70^{\circ} \mathrm{C}$ drying temperature. The maximum average scores for colour, texture, taste, appearance and overall acceptability were found as 7.6, 7.2, 6.9, 7.2 and 7.2 for yam chips. The crispiness of the best sample of fried yam chips was found comparable with branded potato chips available in market. Thus, it could be recommended that the prepared dehydrated yam slices as well as yam chips were of very good quality and could be stored for 4 months in polythelene pack (200 gauge). Value added products could very well be prepared without much extra investment at home scale level like potato chips.

- KEY WORDS : Yam, Pre-treatment, Dehydrated chips, Rehydration, Sensory evaluation, Storage

- HOW TO CITE THIS PAPER : Kumar, Arvind, Nath, Alok and Sharma, Pratibha Devi (2018). Development of process technology for the preparation of yam (Amorphophallus spp.) chips. Internat. J. Agric. Engg., 11(1) : 184-189, DOI: 10.15740/HAS/IJAE/11.1/184-189. 